\title{
Analyzing Time-to-Market and Reliability Trade-Offs with Bayesian Belief Networks
}

\author{
Jianyun Zhou and Tor Stålhane \\ Department of Computer and Information Science, \\ Norwegian University of Science and Technology, 7491 Trondheim, Norway \\ \{jianyun, stalhane\}@idi.ntnu.no
}

\section{Introduction}

Web-based systems are software systems with a Web interface. In recent years it has successfully penetrated into our everyday life. Development of such systems has also opened a new area in software engineering: Web engineering. Many studies found that Web development often experiences much shorter cycle time than traditional software development. It conflicts with some non-functional requirements, such as system reliability. When they can not be simultaneously satisfied, trade-off decisions must be made. Such decisions are typically difficult in software development due to the complexity and uncertainty of the development process. They are usually decided in an ad hoc manner. The purpose of this paper is to propose a new methodology for conducting trade-off analysis. Bayesian Belief Networks (BBNs) is used to offer this opportunity. By capturing and modelling the cause-effect relationships between time and reliability in BBN models, it provides a systematic way for doing comprehensive trade-off analysis throughout the whole development process.

\section{Why Bayesian Belief Network (BBN)}

A $\mathrm{BBN}$ is a directed graph, together with an associated set of probability tables to express cause-effect relationships between linked nodes. The use of BBN in software engineering concerns mostly software quality. Recently its application extends to other areas, such as process modeling and cost estimation. Our objective is to use it as a tool to conduct trade-off analysis for time-to-market and reliability. Analyzing the relationships between time-to-market and reliability in the development process is not an easy task: (1) their relationship is not explicit and can not be directly identified. It is transferred by the development process and activities in the process; (2) their relationship is uncertain. There are a lot of confounding factors existing in the process; (3) their relationship is not easy to be evidenced. Different kinds of data from difference sources are difficult to be combined together.

Regarding the advantages of BBNs, the above mentioned problems can be solved because: (1) BBNs are able to model the development process and activities in the process by depicting the cause-effect relationship between the attributes of process components; (2) BBNs identify probabilistic relationships among nodes. They enable 
to reason with uncertain and incomplete information by adding a probability to both the input and output information; (3) BBNs can collect evidence from many different sources, such as expert knowledge, historical data, and so on.

In general, with BBNs we can capture direct and indirect, explicit and implicit relationships between time-to-market and reliability throughout the development process. Such models will support trade-off analysis to be performed in a process scale.

\section{Trade-Off Analysis Model for Web-Based System Development}

The overall goal of the model is to facilitate trade-off analysis for time-to-market and reliability. Particularly, the model is supposed to address how the total development time and time allocation will affect the reliability of the final system. It will help decision makers choose appropriate time strategies for achieving reliability requirements, and provide a foundation for schedule planning and progress control.

Developing such a model involves three steps: (1) Identify process components, that is, main activities involved in the development; (2) Identifying attributes of the process components, which are relevant to trade-off analysis; (3) Identifying probabilistic dependencies between attributes and draw cause-effect diagram.

For the purpose of illustration, we develop a generic model based on a general understanding of activities involved in the Web development process. At first we define that any Web-based system development consists of the following activities: Requirements and analysis; Architecture design; System development; and Testing. Each of activities has two sub-phases: initial work and rework. The purpose of this decomposition is closely related to the goal of trade-off analysis as how the time is allocated to these activities will affect the system reliability. In addition, reliability is not only affected by the development process, it is also a property of the system. Web-based system is usually constructed using multiple modules. The activity component system development can therefore be further decomposed into sub-activities for developing presentation layer, business logic layer, and data management layer. How the time is allocated to develop these layers will have effects on the system reliability.

The full model is illustrated in Figure 1. It consists of fragments that represent different process components. In requirements and analysis fragment, the node Problem complexity represents the degree of complexity inherent in the new system to be developed. It influences the actual time used in the requirements initiative and the serious defects introduced in the initial requirements documents. During requirements rework, a number of the serious defects are found and fixed. The node defects remained after requirements contains the total number of defects inherent at the end of the phase. Fragments architecture design, development and testing follow the same principle. The node Technical risk is defined as level of difficulty under development. It influences both the time attribute and the introduced defects attribute. Finally, the reliability level of the system is directly influenced by Defects in presentation layer, Defects in business logic layer, Defects in data management layer, and Defects delivered. 


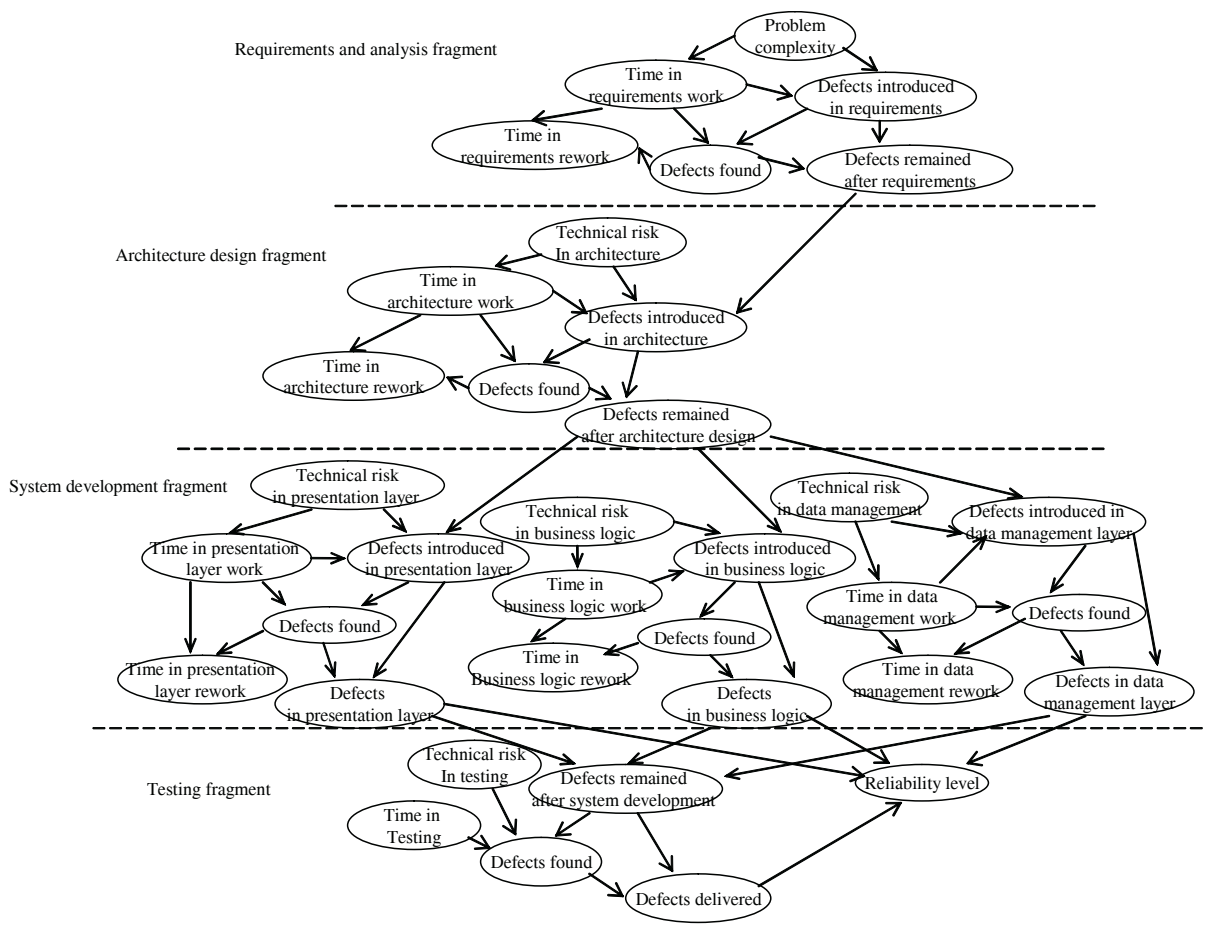

Fig. 1. BBN process model for trade-off analysis

\section{Discussion}

The effect of time allocation on reliability is transferred by variables in the model. It provides us an opportunity to perform trade-off analysis for time-to-market and reliability and gives decision makers an objective basis for assessing different time strategies, predicting reliability, and controlling progress. We do not make any claims about this model being correct for all situations. In particular, we have not taken into account some typical features of the process, such as iteration, increments, and parallelism. As these properties influence development time and introduced defects, they should be captured in proper variables. The detailed level of attributes in the model is also very limited. Many other attributes might be included in the topology. The exact process variables and topology are context-dependent.

Although a BBN model is easy to build, the real difficulty lies in the validation, that is, determining whether the model correctly represent the relationships in a real process. At the heart of the problem lies a lack of quantitative data. It seems that we have little choice but to rely on information from domain experts. How to collect and treat with this information is therefore a critical issue for the refinement of the BBN analysis model. It is also one of our main research interests in the future. 